\title{
Previous History of Knee Arthroscopy in Patients Undergoing Total Knee Arthroplasty: An Examination of the Effect of the Literature and American Academy of Orthopaedic Surgeons 2013 Arthroscopy Guidelines on Clinical Practice
}

\author{
Melissa A. Kluczynski, M.S.., Griffin Lunn, Matthew J. Phillips, M.D., and \\ John M. Marzo, M.D.
}

\begin{abstract}
Purpose: To compare the rate of previous knee arthroscopy in patients undergoing total knee arthroplasty (TKA) before (2005-2006) and after (2018) publication of landmark studies that examined the effectiveness of knee arthroscopy as well as the American Academy of Orthopaedic Surgeons 2013 knee arthroscopy guidelines. Methods: We performed a retrospective chart review of 214 patients who underwent a TKA between 2005 and 2006 (Group 1) and 213 patients who underwent a TKA in 2018 (Group 2). We reviewed medical records to determine whether previous knee arthroscopy was performed. Group comparisons were made using the $t$ test for continuous data and the $\chi^{2}$ test for categorical data, and means \pm standard deviation are presented. Results: Patients in Group 2 were older than those in Group 1 (67.0 \pm 8.3 vs $64.3 \pm 11.5$ years, $P=.01)$; however, there were no differences between groups with respect to sex $(P=.40)$, body mass index $(P=.36)$, and laterality of TKA $(P=.05)$. There was no difference in the history of previous knee arthroscopy between Groups 1 and 2 overall $(27 \%$ vs $32 \%, P=.27)$ and within 10 years of TKA $(17 \%$ vs $15 \%, P=.44)$. Conclusions: We did not find a difference in the number of previous knee arthroscopies for patients undergoing TKA in 2018 versus 2005-2006. This suggests that the recommendations set forth by landmark clinical trials that examined the effectiveness of knee arthroscopy and the AAOS 2013 knee arthroscopy guidelines did not have a sudden impact within our geographic region, but there is some evidence to suggest a gradual shift in treatment, where knee arthroscopy is withheld near imminent knee arthroplasty. Level of Evidence: III, retrospective comparative study.
\end{abstract}

A bout 14 million people in the United States suffer from symptomatic knee osteoarthritis (OA), resulting in pain and loss of function and activity. ${ }^{1,2}$ Symptoms initially are managed conservatively (e.g., physical therapy), although no treatment currently exists that alters the structural progression of

From the Jacobs School of Medicine and Biomedical Sciences, University at Buffalo, State University of New York, Buffalo, New York, U.S.A.

The authors report the following potential conflicts of interest or sources of funding: This study was funded by the Ralph C. Wilson, Jr. Foundation. Full ICMJE author disclosure forms are available for this article online, as supplementary material.

Received November 11, 2020; accepted February 18, 2021

Address correspondence to Melissa A. Kluczynski, M.S., 4949 Harlem Rd., Amherst, NY14226.E-mail:mk67@buffalo.edu.

(C) 2021 THE AUTHORS. Published by Elsevier Inc. on behalf of the Arthroscopy Association of North America. This is an open access article under the CC BY-NC-ND license (http://creativecommons.org/licenses/by-nc-nd/4.0/). 2666-061X/201840

https://doi.org/10.1016/j.asmr.2021.02.006 knee OA. When conservative management fails, knee arthroscopy or total knee arthroplasty (TKA) is considered for management of continued pain and disability. However, the efficacy of knee arthroscopy for OA was challenged after Moseley et al.'s landmark randomized controlled trial in 2002. ${ }^{3}$ Moseley et al. ${ }^{3}$ found no difference in clinical outcomes following arthroscopic debridement or arthroscopic lavage in comparison with placebo among patients with knee OA. Soon thereafter, in 2008, Kirkley et al. ${ }^{4}$ found no difference in outcomes for arthroscopic lavage and debridement plus physical and medical therapy versus physical and medical therapy alone in patients with knee OA. Based largely on these studies, the American Academy of Orthopaedic Surgeons (AAOS) published evidence-based practice guidelines in 2013 stating that arthroscopy with lavage and/or debridement in patients with a primary diagnosis of symptomatic knee OA could not be recommended. ${ }^{5}$ In general, however, with 
respect to the nonarthroplasty management of $\mathrm{OA}$, there is only modest adherence to the recommendations contained within the AAOS clinical practice guidelines. $^{6}$

Many patients undergoing knee arthroscopy eventually go on to have TKA. One systematic review estimated the annual incidence of TKA by dividing the cumulative incidence (number of TKAs among those who underwent arthroscopy divided by the total number of patients who had arthroscopy) by the mean years of follow-up. ${ }^{2}$ Using data from 20 studies, this review estimated the annual incidence of TKA to be $2.6 \%$ following knee arthroscopy in patients with knee OA. In a study by Camus et al., ${ }^{7}$ the incidence of TKA was $3.9 \%$ at an average of 3 years after knee arthroscopy for OA. Despite published practice guidelines, research has shown noncompliance with proper use of knee arthroscopy by clinical practitioners. ${ }^{8}$ Some studies have investigated the effect of Moseley et al.'s findings on clinical practice. ${ }^{9-12}$ Holmes et al. ${ }^{9}$ found a $49 \%$ decrease in knee arthroscopies performed in the state of Florida between 2000 and 2008 that was attributed to changes in reimbursement and lack of efficacy of knee arthroscopy for treating knee OA. In a study by Potts et al., ${ }^{3,10}$ the total number of knee arthroscopy cases per surgeon decreased from a high of 11.9 in 2003 to a low of 8.6 in 2009, which the authors attributed to publication of the study by Moseley et al. Harris et al. ${ }^{12}$ found that the rates of knee arthroscopy generally remained steady between 2000 and 2008, and the rate of TKA declined among patients aged 65 and older. However, none of these studies examined whether the rate of knee arthroscopies for OA decreased before TKA, which is essential since previous knee arthroscopy may result in worse outcomes after TKA. $^{13}$

The purpose of this study was to compare the rate of previous knee arthroscopy in patients undergoing TKA before (2005-2006) and after (2018) publication of landmark studies ${ }^{3,4}$ that examined the effectiveness of knee arthroscopy as well as the AAOS 2013 knee arthroscopy guidelines. We hypothesized that there would be a difference in the rate of previous knee arthroscopy between 2005 and 2006 and 2018.

\section{Methods}

We performed a retrospective chart review to identify TKAs performed by a single surgeon at our institution from January 2005-December 2006 and January 2018December 2018. We included patients who had knee arthroscopy at any time before TKA. Billing records were searched for the Common Procedural Terminology code 27447, and a total of 507 records were identified. It should be noted that the study surgeon did not perform any of the previous knee arthroscopies identified by this study. Based on our sample size calculation, we included the first 214 patients in our records that underwent a TKA between 2005-2006 (Group 1) and the first 213 patients that underwent a TKA in 2018 (Group 2). The remaining 80 records were excluded. Two trained research assistants searched each patient's medical records to determine whether a previous knee arthroscopy was performed either at our institution or elsewhere. Demographic data were also collected, including age, sex, body mass index, and laterality. An institutional review board approved this study (IRB \#STUDY00004618).

Descriptive statistics were calculated for demographic data and for the history of previous knee arthroscopy. Previous knee arthroscopy was categorized as yes/no, and also the actual number of previous knee arthroscopies was quantified. The average number of previous knee arthroscopies was calculated for (1) the entire sample who had at least 1 knee arthroscopy and (2) for patients who had at least 1 arthroscopy within 10 years of TKA. The $t$-test was used for comparing continuous data and the $\chi^{2}$ test for comparing categorical data between Groups 1 and 2. We compared previous knee arthroscopies between Groups 1 and 2, both overall and specifically within 10 years of TKA.

Table 1. Demographics by Study Group

\begin{tabular}{|c|c|c|c|}
\hline & $\begin{array}{c}\text { Group } 1 \text { (TKA Performed } \\
\text { in } 2005-2006, \mathrm{~N}=214 \text { ) }\end{array}$ & $\begin{array}{c}\text { Group } 2 \text { (TKA Performed } \\
\text { in } 2018, \mathrm{~N}=213 \text { ) }\end{array}$ & $P$ Value ${ }^{*}$ \\
\hline Age, y & $64.3 \pm 11.46$ & $67.0 \pm 8.31$ & .01 \\
\hline Body mass index & $34.03 \pm 8.65^{\dagger}$ & $33.31 \pm 7.56$ & .36 \\
\hline Sex & & & .40 \\
\hline Male & $70(32.7 \%)$ & $78(36.6 \%)$ & \\
\hline Female & $144(67.3 \%)$ & $135(63.4 \%)$ & \\
\hline TKA laterality & & & .05 \\
\hline Right & $88(41.1 \%)$ & $91(42.7 \%)$ & \\
\hline Left & $67(31.3 \%)$ & $84(39.4 \%)$ & \\
\hline Bilateral & $59(27.6 \%)$ & $38(17.8 \%)$ & \\
\hline
\end{tabular}

NOTE. Data are reported as mean \pm standard deviation or $\mathrm{N}(\%)$.

TKA, total knee arthroplasty.

*The $P$ value was derived from $t$-tests for comparisons of continuous data and $\chi^{2}$ test for comparisons of categorical data.

${ }^{\dagger}$ Body mass index was missing for 2 patients in Group 1 and for 1 patient in Group 2. 
Table 2. Frequency of Previous Arthroscopy by Study Group

\begin{tabular}{lcr}
\hline & $\begin{array}{c}\text { Group 1 (TKA Performed } \\
\text { in 2005-2006) }\end{array}$ & $\begin{array}{c}\text { Group 2 (TKA Performed } \\
\text { in 2018) }\end{array}$ \\
\hline $\begin{array}{l}\text { Any previous knee scope } \\
\quad \text { Yes }\end{array}$ & $57(26.6 \%)$ & $67(31.5 \%)$ \\
No & $157(73.36 \%)$ & $146(68.5 \%)$ \\
Previous knee scope within 10 years of TKA ${ }^{\dagger}$ & $37(17.3 \%)$ & .27 \\
$\quad$ Yes & $177(82.7 \%)$ & $31(14.6 \%)$ \\
No & & $182(85.5 \%)$ \\
\hline
\end{tabular}

TKA, total knee arthroplasty

${ }^{*} P$ values were derived from $\chi^{2}$ test.

${ }^{\dagger}$ Categories for the timing of previous knee arthroscopy are not mutually exclusive. Patients may have had a history of multiple knee arthroscopies.

A sample size calculation was done a priori. Based on expected prevalence rates of previous knee arthroscopy of $3.3 \%$ in Group 1 and $2 \%$ in Group 2 and $80 \%$ power, at least 180 patients were needed for each group. All data analyses were performed with SAS 9.4 (SAS Institute, Cary, NC).

\section{Results}

As shown in Table 1, subjects in Group 2 were older than Group $1(P=.01)$, and there were no group differences in body mass index $(P=.36)$, sex $(P=.40)$, and laterality $(P=.05)$. As shown in Table 2 and Figures 1-2, there was no difference in the history of previous knee arthroscopy between Groups 1 and 2 overall $(27 \%$ vs $32 \%, P=.27)$ and within 10 years of
TKA ( $17 \%$ vs $15 \%, P=.44)$. Thirty-two of $427(8 \%)$ patients had multiple previous knee arthroscopies. Among patients who had at least 1 knee arthroscopy, the average number of previous knee arthroscopies was $1.30 \pm 0.53$ for Group 1 and $1.29 \pm 0.55$ for Group 2 . The average number of previous knee arthroscopies also was similar when restricted to patients who had at least 1 knee arthroscopy within 10 years of TKA (Group $1[\mathrm{~N}=37]$ : $1.41 \pm 0.60$; Group $2[\mathrm{~N}=31]$ : $1.23 \pm 0.43)$. Overall, 32 of $427(8 \%)$ patients had multiple previous knee arthroscopies.

\section{Discussion}

The studies by Moseley et al. ${ }^{3}$ and Kirkley et al. ${ }^{4}$ were 2 of the earliest clinical trials to demonstrate that

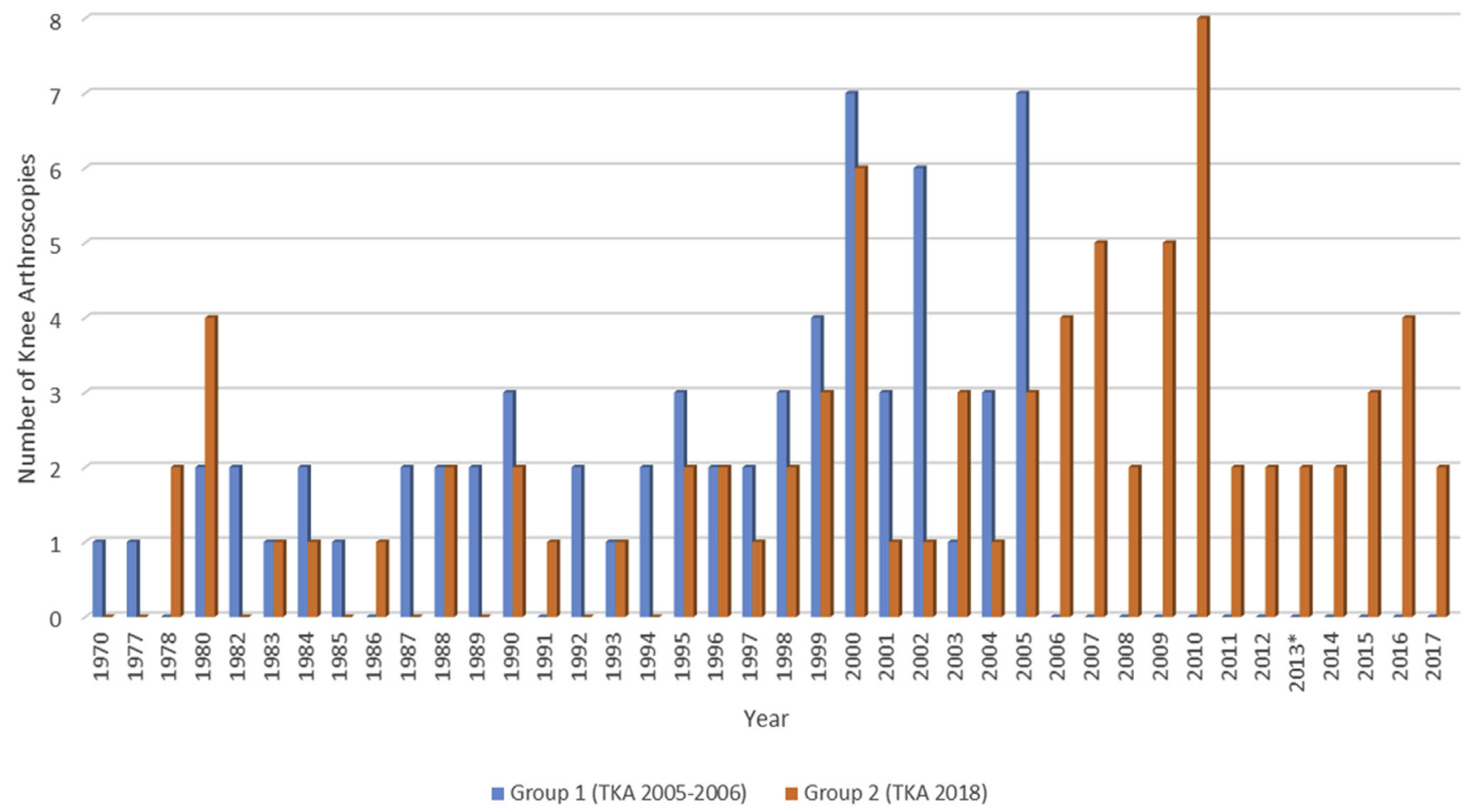

Fig 1. Number of knee arthroscopies before total knee arthroplasty (TKA) by group. *2013 AAOS knee arthroscopy guidelines. Data are not mutually exclusive, since some patients had multiple knee arthroscopies. 


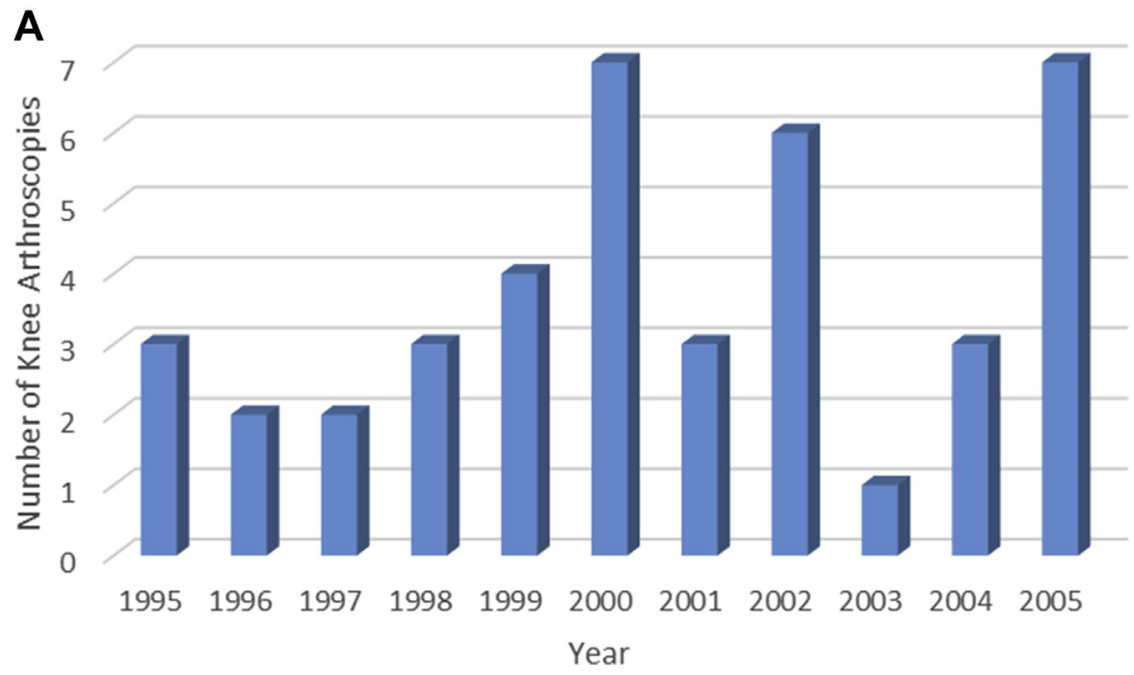

B

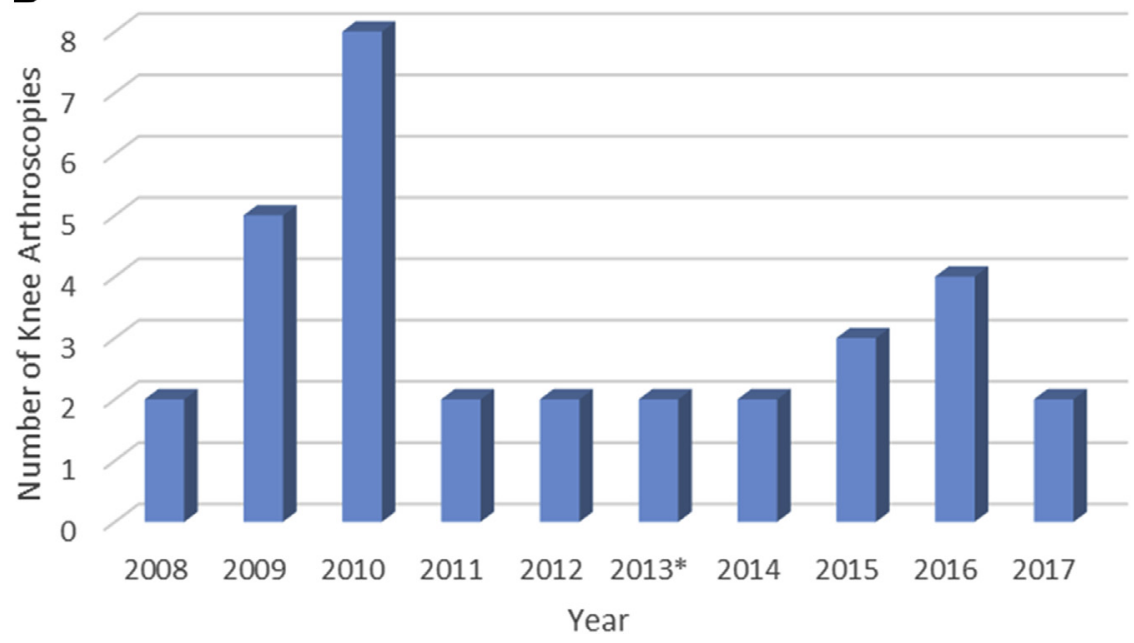

Fig 2. (A) Number of knee arthroscopies before total knee arthroplasty (TKA) within 10 years of TKA for Group 1 (TKA 2005-2006). Data are not mutually exclusive (41 knee arthroscopies in 37 patients). (B) Number of knee arthroscopies before total knee arthroplasty (TKA) within 10 years of TKA for Group 2 (TKA 2018). Data are not mutually exclusive (32 knee arthroscopies in 31 patients). arthroscopic debridement was no better than sham surgery or physical therapy for treating knee OA. In 2013, the AAOS published evidence-based guidelines stating that arthroscopy with lavage and/or debridement in patients with a primary diagnosis of symptomatic knee OA is not recommended. ${ }^{5}$ We examined the effectiveness of the recommendations set forth by these trials and guidelines by comparing the rate of previous knee arthroscopy in patients undergoing TKA before (2005-2006) and after (2018) publication of these guidelines. Contrary to our hypothesis, we found no statistically significant difference in history of previous knee arthroscopy for patients undergoing TKA in 2005-2006 compared with 2018 by a single surgeon. Like other components of the AAOS clinical practice guidelines, our findings call into question adherence to the guidelines related to knee arthroscopy. ${ }^{6}$ Some reasons for nonadherence have been proposed, and include being unaware of the guidelines, conflicting evidence, evidence insufficient to alter practice patterns, time and patient pressures, and limited other options available. 8,14

We found no statistically significant difference in the prevalence of previous knee arthroscopy within 10 years of TKA done in 2005-2006 compared with 2018. Interestingly, we observed that among patients who had a TKA in 2005-2006 previous knee arthroscopy was more often performed closer to the time of TKA (2000-2005), and among patients who had a TKA in 2018, previous knee arthroscopy was more often performed further from the time of TKA (Fig 1). This might suggest that surgeons, in response to the practice guidelines, are gradually withholding knee arthroscopy from patients in whom knee arthroplasty is imminent. However, we only collected data from one arthroplasty surgeon, which limits the generalizability of the results and thus further research is warranted. The study by Moseley et al. was the first landmark trial demonstrating that knee arthroscopy was not effective for treating knee OA. ${ }^{3}$ Potts et al. ${ }^{10}$ found that knee 
arthroscopy as a percentage of total orthopaedic surgery decreased from $10 \%$ in 2003 to $6.6 \%$ in 2009 after the Moseley trial. Furthermore, a Swedish study found a decrease in knee arthroscopies after the national health authority recommended against the use of knee arthroscopy in patients aged 40 years and older with knee OA in 2012. ${ }^{15}$ However, neither of these studies examined whether the rate of knee arthroscopy for OA decreased before TKA. ${ }^{3,15}$ Obtaining a better understanding of the trends in knee arthroscopy and conversion to TKA is important because knee arthroscopy has been shown to be a risk factor for poor outcomes after TKA. For instance, a study by Barton et al. ${ }^{13}$ found that Oxford Knee Scores were worse for patients undergoing TKA that had a knee arthroscopy within the past 6 months.

We found that $17 \%$ of patients that had a TKA in 2005-2006 and 15\% of patients that had a TKA in 2018 also had a history of previous knee arthroscopy within 10 years of TKA. Previous research has found similar rates of conversion to TKA, but no studies have directly compared these rates before and after publication of the AAOS guidelines. Two studies found that $9 \%$ to $11 \%$ of patients undergoing knee arthroscopy progressed to TKA, and increased age was a contributing factor for conversion. ${ }^{16,17}$ Johanson et al. ${ }^{18}$ examined a large Medicare database of more than 78,000 cases that underwent knee arthroscopy and found that 10\% progressed to TKA within 1 year. Boyd and Gradisar ${ }^{19}$ found that the incidence of conversion to TKA increased from $10 \%$ at 1 year to $16 \%$ at 3 years after arthroscopic partial meniscectomy, chondroplasty or debridement in patients older than 50 years old. Risk factors for conversion in this study were age older than 70 years, white race, female sex, obesity, diabetes, nicotine dependence, depression, and rheumatoid arthritis. Another strong predictor of conversion to TKA is advanced radiographic knee OA (i.e., Kellgren-Lawrence Grade 4). ${ }^{20-23}$

\section{Limitations}

There are several limitations of this study. We reviewed patient medical records to determine whether a previous knee arthroscopy was performed either at our institution or elsewhere. It is possible that we were not able to capture all previous surgeries, particularly if they were performed at an outside institution. Furthermore, the timing of surgeries performed outside our institution is subject to patient recall which may be inaccurate. We only included arthroplasty procedures performed by a single surgeon at our institution, and thus our results might not be generalizable to orthopedic surgeons at other institutions. However, based on review of postal zip codes, the vast majority of patients were from the local community as opposed to out-oftown referrals. Furthermore, the study surgeon did not perform any of the arthroscopies before TKA, nor were they involved in the decision-making process for patients included in the current study that underwent arthroscopy. We were not able to discern the reason for knee arthroscopy and thus OA might not have been the primary indication for this procedure. However, we performed a subanalysis of knee arthroscopies done within 10 years of TKA as this was deemed the most likely timeframe for conversion to TKA. Also, the study groups may not have been entirely comparable since patients who had TKA in 2018 were slightly older than those who had TKA in 2005-2006. This may reflect the surgeon's preference (M.J.P.) to delay TKA for at least 1 year after arthroscopy, however selection for TKR by the arthroplasty surgeon did not affect the decision for arthroscopy.

\section{Conclusions}

We did not find a difference in the number of previous knee arthroscopies for patients undergoing TKA in 2018 versus $2005-2006$. This suggests that the recommendations set forth by landmark clinical trials and the AAOS 2013 guidelines did not have a sudden impact within our geographic region, but there is some evidence to suggest a gradual shift in treatment, where knee arthroscopy is withheld near imminent knee arthroplasty.

\section{Acknowledgments}

We acknowledge Kathleen Lafferty for her assistance with collecting and verifying data for this study.

\section{References}

1. Deshpande BR, Katz JN, Solomon DH, et al. Number of persons with symptomatic knee osteoarthritis in the US: Impact of race and ethnicity, age, sex, and obesity. Arthritis Care Res (Hoboken) 2016;68:1743-1750.

2. Winter AR, Collins JE, Katz JN. The likelihood of total knee arthroplasty following arthroscopic surgery for osteoarthritis: A systematic review. BMC Musculoskelet Disord 2017;18:408.

3. Moseley JB, O'Malley K, Petersen NJ, et al. A controlled trial of arthroscopic surgery for osteoarthritis of the knee. N Engl J Med 2002;347:81-88.

4. Kirkley A, Birmingham TB, Litchfield RB, et al. A randomized trial of arthroscopic surgery for osteoarthritis of the knee. N Engl J Med 2008;359:1097-1107.

5. American Academy of Orthopaedic Surgeons. Treatment of osteoarthritis of the knee: Evidence-based guideline, 2nd ed. Rosemont, IL: American Academy of Orthopaedic Surgeons, 2013.

6. Meiyappan KP, Cote MP, Bozic KJ, Halawi MJ. Adherence to the American Academy of Orthopaedic Surgeons Clinical Practice Guidelines for Nonoperative Management of Knee Osteoarthritis. J Arthroplasty 2020;35: 347-352. 
7. Camus T, Han J, Osmani F, Scott N, Long WJ. Incidence and predictors of total knee arthroplasty following knee arthroscopy. J Orthop 2018;15:32-35.

8. Carlson VR, Ong AC, Orozco FR, Hernandez VH, Lutz RW, Post ZD. Compliance With the AAOS guidelines for treatment of osteoarthritis of the Knee: A survey of the American Association of Hip and Knee Surgeons. J Am Acad Orthop Surg 2018;26:103-107.

9. Holmes R, Moschetti W, Martin B, Tomek I, Finlayson S. Effect of evidence and changes in reimbursement on the rate of arthroscopy for osteoarthritis. Am J Sports Med 2013;41:1039-1043.

10. Potts A, Harrast JJ, Harner CD, Miniaci A, Jones MH. Practice patterns for arthroscopy of osteoarthritis of the knee in the United States. Am J Sports Med 2012;40:1247-1251.

11. Adelani MA, Harris AH, Bowe TR, Giori NJ. Arthroscopy for knee osteoarthritis has not decreased after a clinical trial. Clin Orthop Relat Res 2016:474:489-494.

12. Harris IA, Madan NS, Naylor JM, Chong S, Mittal R, Jalaludin BB. Trends in knee arthroscopy and subsequent arthroplasty in an Australian population: a retrospective cohort study. BMC Musculoskelet Disord 2013;14:143.

13. Barton SB, McLauchlan GJ, Canty SJ. The incidence and impact of arthroscopy in the year prior to total knee arthroplasty. Knee 2017;24:396-401.

14. Barlow T, Plant CE. Why we still perform arthroscopy in knee osteoarthritis: A multi-methods study. BMC Musculoskelet Disord 2015;16:85.

15. Kiadaliri A, Bergkvist D, Dahlberg LE, Englund M. Impact of a national guideline on use of knee arthroscopy: An interrupted time-series analysis. Int J Qual Health Care 2019;31:G113-G118.
16. Bernard J, Lemon M, Patterson MH. Arthroscopic washout of the knee-a 5-year survival analysis. Knee 2004; 11:233-235.

17. Jameson SS, Rushton SP, Dowen D, et al. Increasing age and female gender are associated with early knee replacement following arthroscopy. Knee Surg Sports Traumatol Arthrosc 2014;22:2665-2671.

18. Johanson NA, Kleinbart FA, Cerynik DL, Brey JM, Ong KL, Kurtz SM. Temporal relationship between knee arthroscopy and arthroplasty: A quality measure for joint care? J Arthroplasty 2011;26:187-191.

19. Boyd JA, Gradisar IM. Total knee arthroplasty after knee arthroscopy in patients older than 50 years. Orthopedics 2016;39:e1041-e1044.

20. Steadman JR, Briggs KK, Matheny LM, Ellis HB. Ten-year survivorship after knee arthroscopy in patients with Kellgren-Lawrence grade 3 and grade 4 osteoarthritis of the knee. Arthroscopy 2013;29:220-225.

21. Zikria B, Hafezi-Nejad N, Wilckens J, Ficke JR, Demehri S. Determinants of knee replacement in subjects with a history of arthroscopy: Data from the osteoarthritis initiative. Eur J Orthop Surg Traumatol 2016;26:665-670.

22. Raaijmaakers M, Vanlauwe J, Vandenneucker $H$, Dujardin J, Bellemans J. Arthroscopy of the knee in elderly patients: Cartilage lesions and their influence on short term outcome. A retrospective follow-up of 183 patients. Acta Orthop Belg 2010;76:79-85.

23. Hawker G, Guan J, Judge A, Dieppe P. Knee arthroscopy in England and Ontario: Patterns of use, changes over time, and relationship to total knee replacement. J Bone Joint Surg Am 2008;90:2337-2345. 